\title{
QUASIADDITIVITY AND MEASURE PROPERTY OF CAPACITY AND THE TANGENTIAL BOUNDARY BEHAVIOR OF HARMONIC FUNCTIONS
}

\author{
H. AIKAWA AND A. A. BORICHEV
}

Dedicated to Professor F.-Y. Maeda on the occasion of his sixtieth birthday

Abstract. We show that if a set $E$ is dispersely decomposed into subsets, then the capacity of $E$ is comparable to the summation of the capacities of the subsets. From this fact it is derived that the Lebesgue measure of a certain expanded set is estimated by the capacity of $E$. These properties hold for classical capacities, $L^{p}$-capacities and energy capacities of general kernels. The estimation is applied to the boundary behavior of harmonic functions. We introduce a boundary thin set and show a fine limit type boundary behavior of harmonic functions. We show that a thin set does not meet essentially Nagel-Stein and Nagel-Rudin-Shapiro type approaching regions at almost all bounary points.

\section{IntRoduction}

Let $K(r) \not \equiv 0$ be a nonnegative nonincreasing lower semicontinuous (l. s. c.) function for $r>0$. We assume that

$$
\lim _{r \rightarrow 0} K(r)=\infty \text { and } \lim _{r \rightarrow \infty} K(r)=0
$$

For $x \in \mathbb{R}^{N}$ we define $K(x)=K(|x|)$, and assume that $K(x)$ is locally integrable on $\mathbb{R}^{N}$, in other words

$$
\int_{0} K(t) t^{N-1} d t<\infty
$$

We define the capacity $C_{K}$ by

$$
C_{K}(E)=\inf \{\|\mu\|: K * \mu \geq 1 \text { on } E\} .
$$

Let $K_{\alpha}(r)=r^{\alpha-N}$ for $0<\alpha<N$. This is the Riesz kernel of order $\alpha$. If $K(r)=K_{\alpha}(r)$, then we write $C_{\alpha}$ for $C_{K}$ and call it $\alpha$-capacity.

Received by the editors April 25, 1994

1991 Mathematics Subject Classification. Primary 31B15, 31B25.

Key words and phrases. Quasiadditivity of capacity, boundary behavior of harmonic functions, thin set, fine limit, approach region.

This work was started when the first author visited the Department of Mathematics, University of Uppsala. He acknowledges support from the Royal Swedish Academy of Sciences and the Japan Society of Promotion of Science. 
Let $h(r)$ be a positive nondecreasing function for $r>0$ and $h(0)=0$. Such a function is called a measure function. We define the content $M_{h}$ by

$$
M_{h}(E)=\inf \left\{\sum h\left(r_{j}\right): E \subset \bigcup B\left(x_{j}, r_{j}\right)\right\},
$$

where $B(x, r)$ stands for the open ball with center at $x$ and radius $r$. If $h(r)=r^{\beta}$, then we write $M_{\beta}$ for $M_{h}$ and call it $\beta$-content.

It is well known that the capacity $C_{\alpha}$ and the content $M_{\beta}$ are closely related:

(i) If $\beta>N-\alpha$, then $C_{\alpha}(E)=0 \Longrightarrow M_{\beta}(E)=0$.

(ii) If $\beta \leq N-\alpha$, then $M_{\beta}(E)=0 \Longrightarrow C_{\alpha}(E)=0$.

Since $C_{\alpha}$ is countably subadditive and homogeneous of degree $N-\alpha$, it follows that

$$
C_{\alpha}(E) \leq A M_{N-\alpha}(E)
$$

which readily yields (ii). It is noteworthy that we cannot put $\beta=N-\alpha$ in (i); there is a set $E$ such that $C_{\alpha}(E)=0$ and $M_{N-\alpha}(E)>0$. Thus the $C_{\alpha}$-capacity does not majorize the $(N-\alpha)$-dimensional measure.

One of the main purposes of this paper is to compare $C_{\alpha}$ with a certain $(N-\alpha)$ dimensional quantity. Hereafter we shall use the following notation. By the symbol $A$ we denote an absolute positive constant whose value is unimportant and may change from line to line. We shall say that two positive quantities $f$ and $g$ are comparable, written $f \approx g$, if and only if there exists a constant $A$ such that $A^{-1} g \leq f \leq A g$. By $|E|$ we denote the Lebesgue measure of $E$. For a proper subset $E$ we put $\delta_{E}(x)=\operatorname{dist}\left(x, E^{c}\right)$. Let us consider the set

$$
\widetilde{E}_{\alpha}=\bigcup_{x \in E} B\left(x, \delta_{E}(x)^{(N-\alpha) / N}\right) .
$$

It is easy to see that if $E=B(0, r)$ and $r>0$ is small, then $\widetilde{E}_{\alpha}$ is a ball with radius comparable to $r^{(N-\alpha) / N}$, so that

$$
\left|\widetilde{E}_{\alpha}\right| \approx r^{N-\alpha} \text { for small } r>0 .
$$

Thus $\left|\widetilde{E}_{\alpha}\right|$ is, in a sense, an $(N-\alpha)$-dimensional quantity. We shall show the following theorem.

Theorem 1. Let $U$ be a bounded set. Suppose $E \subset U$ and let $\widetilde{E}_{\alpha}$ be as above. Then

$$
\left|\widetilde{E}_{\alpha}\right| \leq A C_{\alpha}(E)
$$

where $A>0$ depends only on $N, \alpha$ and $U$.

Let $g_{\alpha}$ be the Bessel kernel. The Riesz and the Bessel kernels have the same asymptotics as $r \rightarrow 0$. However, $g_{\alpha}(r)$ decreases rapidly as $r \rightarrow \infty$ and hence $g_{\alpha}$ is integrable on $\mathbb{R}^{N}$. It is well known that

$$
C_{\alpha}(E) \approx C_{g_{\alpha}}(E) \text { for } E \subset U,
$$

where $U$ is a bounded set. Hence Theorem 1 follows from Theorem 2 below. Let us consider the capacity of a ball. Suppose $K * \mu \geq 1$ on $B(0, r)$. Then $|B(0, r)| \leq$ $\|K\|_{1}\|\mu\|$ by Fubini's theorem, so that

$$
C_{K}(B(0, r)) \geq\|K\|_{1}^{-1}|B(0, r)|
$$


by definition. We define a positive function $\eta(r)$ by $|B(0, \eta(r))|=C_{K}(B(0, r))$ and let $\eta^{*}(r)=\max \{\eta(r), 2 r\}$. It follows from (1) that

$$
\eta(r) \approx \eta^{*}(r) \geq A r
$$

We put

$$
\widetilde{E}_{K}=\bigcup_{x \in E} B\left(x, \eta^{*}\left(\delta_{E}(x)\right)\right) .
$$

This is a set enlarged from $E$ if $E$ is open.

Theorem 2. Let $K$ be an integrable kernel. Then

$$
\left|\widetilde{E}_{K}\right| \leq A C_{K}(E),
$$

where $A>0$ depends only on $N$ and $K$.

Theorem 2 comes from the following quasiadditive property of $C_{K}$ : if a set $E$ is decomposed into pieces $E_{j}$ dispersely, then $C_{K}(E)$ is comparable to $\sum C_{K}\left(E_{j}\right)$. More precisely we have the following theorem.

Theorem 3. Let $K$ be an integrable kernel. Suppose $\left\{B\left(x_{j}, \eta^{*}\left(r_{j}\right)\right)\right\}$ is disjoint and $E$ is an analytic subset of $\bigcup B\left(x_{j}, r_{j}\right)$. Then

$$
C_{K}(E) \leq \sum C_{K}\left(E \cap B\left(x_{j}, r_{j}\right)\right) \leq A C_{K}(E)
$$

where $A>0$ depends only on $N$ and $K$.

Theorem 2 and Theorem 3 have counterparts in $L^{p}$-capacity theory. Let $1<$ $p<\infty$. We define

$$
C_{K, p}(E)=\inf \left\{\|f\|_{p}^{p}: K * f \geq 1 \text { on } E\right\} .
$$

Let us consider the capacity of a ball. Suppose $K * f \geq 1$ on $B(0, r)$. Then $\left\|\chi_{B(0, r)}\right\|_{p} \leq\|K\|_{1}\|f\|_{p}$, so that

$$
C_{K, p}(B(0, r)) \geq\|K\|_{1}^{-p}|B(0, r)|
$$

by definition. We define a positive function $\eta_{p}(r)$ by

$$
\left|B\left(0, \eta_{p}(r)\right)\right|=C_{K, p}(B(0, r))
$$

and let $\eta_{p}^{*}(r)=\max \left\{\eta_{p}(r), 2 r\right\}$. It follows from (3) that

$$
\eta_{p}(r) \approx \eta_{p}^{*}(r) \geq A r .
$$

We put

$$
\widetilde{E}_{K, p}=\bigcup_{x \in E} B\left(x, \eta_{p}^{*}\left(\delta_{E}(x)\right)\right) .
$$


Theorem 4. Let $K$ be an integrable kernel. Then

$$
\left|\widetilde{E}_{K, p}\right| \leq A C_{K, p}(E)
$$

where $A>0$ depends only on $N, K$ and $p$.

Theorem 5. Let $K$ be an integrable kernel. Suppose $\left\{B\left(x_{j}, \eta_{p}^{*}\left(r_{j}\right)\right)\right\}$ is disjoint and $E$ is an analytic subset of $\bigcup B\left(x_{j}, r_{j}\right)$. Then

$$
C_{K, p}(E) \leq \sum C_{K, p}\left(E \cap B\left(x_{j}, r_{j}\right)\right) \leq A C_{K, p}(E)
$$

where $A>0$ depends only on $N, K$ and $p$.

We note that the quasiadditivity in Theorem 3 and Theorem 5 is different from the quasiadditivity considered in [3] and [4]. Theorem 2 and Theorem 4 have an application to the tangential boundary behavior of harmonic functions. We shall later generalize these theorems, in connection with Nagel-Stein approach regions ([8]). We shall introduce a notion of "thin sets" and combine it with the generalized version of Theorem 2 and Theorem 4 to obtain precise description of the tangential boundary behavior of harmonic functions given as the Poisson integral of certain potentials.

The plan of this paper is as follows. We prove Theorem 3 and Theorem 5 in Section 2 and Section 3, respectively. In Section 4 we prove Theorem 2 and Theorem 4. In Section 5 we introduce the Nagel-Stein approach region and generalize Theorem 2 and Theorem 4. The boundary behavior of harmonic function will be considered in Sections 6-10. We shall introduce a new notion of boundary thinness in Section 6.

Hereafter, we let $K$ be an integrable kernel, unless otherwise specified.

\section{Proof of Theorem 3}

We shall need to give a lower estimate for the capacity. To this end we use the dual definition of $C_{K}$.

Theorem A. Let $E$ be an analytic set. Then

$$
C_{K}(E)=\sup \left\{\|\mu\|: \mu \text { is concentrated on } E, K * \mu \leq 1 \text { on } \mathbb{R}^{N}\right\} \text {. }
$$

We prepare an elementary lemma.

Lemma 1. Let $0<2 r \leq R$. Suppose $x \notin B\left(x_{0}, R\right)$ and let $\rho=\operatorname{dist}\left(x, B\left(x_{0}, r\right)\right)$. Then

$$
\left|B(x, \rho) \cap B\left(x_{0}, R\right)\right| \geq A\left|B\left(x_{0}, R\right)\right|,
$$

where $A$ depends only on the dimension.

Proof. Let $x_{1}$ be the point on the line segment connecting $x_{0}$ and $x$ such that $\left|x_{1}-x_{0}\right|=\frac{3}{4} R$. It is easy to see that

$$
\rho=\left|x-x_{0}\right|-r=\left|x-x_{1}\right|+\frac{3}{4} R-r \geq\left|x-x_{1}\right|+\frac{1}{4} R .
$$


We observe that

$$
B\left(x_{1}, \frac{1}{4} R\right) \subset B(x, \rho) \cap B\left(x_{0}, R\right),
$$

since if $y \in\left(x_{1}, \frac{1}{4} R\right)$, then

$$
|x-y|<\left|x-x_{1}\right|+\frac{1}{4} R \leq \rho \text { and }\left|x_{0}-y\right|<\left|x_{0}-x_{1}\right|+\frac{1}{4} R=R .
$$

Thus the required inequality follows.

Proof of Theorem 3. The countable subadditivity yields

$$
C_{K}(E) \leq \sum C_{K}\left(E \cap B\left(x_{j}, r_{j}\right)\right)
$$

Let us prove $\sum C_{K}\left(E \cap B\left(x_{j}, r_{j}\right)\right) \leq A C_{K}(E)$. For simplicity we write $E_{j}$ for $E \cap B\left(x_{j}, r_{j}\right)$. Let $\varepsilon$ be an arbitrary positive number. By Theorem A we can find a measure $\mu_{j}$ such that

$$
\begin{aligned}
& \mu_{j} \text { is concentrated on } E_{j}, \\
& K * \mu_{j} \leq 1 \text { on } \mathbb{R}^{N}, \\
& \left\|\mu_{j}\right\| \geq C_{K}\left(E_{j}\right)-2^{-j} \varepsilon .
\end{aligned}
$$

Let $\mu=\sum \mu_{j}$. Observe that $\mu$ is concentrated on $E$. We claim

$$
K * \mu \leq A \text { on } \mathbb{R}^{N}
$$

If we have (5), then Theorem A yields

$$
C_{K}(E) \geq A\|\mu\|=A \sum\left\|\mu_{j}\right\| \geq A \sum C_{K}\left(E_{j}\right)-\varepsilon .
$$

Since $\varepsilon>0$ is arbitrary, the required inequality follows.

For the proof of (5) we put

$$
d \mu_{j}^{\prime}=\frac{\left\|\mu_{j}\right\|}{\left|B\left(x_{j}, \eta^{*}\left(r_{j}\right)\right)\right|} \chi_{B\left(x_{j}, \eta^{*}\left(r_{j}\right)\right)} d x
$$

and $\mu^{\prime}=\sum \mu_{j}^{\prime}$. This kind of measure was introduced by Borichev [5]. Since

$$
\left\|\mu_{j}\right\| \leq C_{K}\left(E_{j}\right) \leq C_{K}\left(B\left(x_{j}, r_{j}\right)\right)=\left|B\left(x_{j}, \eta\left(r_{j}\right)\right)\right| \leq\left|B\left(x_{j}, \eta^{*}\left(r_{j}\right)\right)\right|,
$$

it follows that

$$
d \mu^{\prime} \leq \sum \chi_{B\left(x_{j}, \eta^{*}\left(r_{j}\right)\right)} d x \leq d x,
$$

where we have used the disjointness of $B\left(x_{j}, \eta^{*}\left(r_{j}\right)\right)$ in the last inequality. Hence

$$
K * \mu^{\prime} \leq K * 1=\int_{\mathbb{R}^{N}} K d x<\infty \text { on } \mathbb{R}^{N}
$$


Let us compare $K * \mu$ and $K * \mu^{\prime}$. Suppose first $x \notin B\left(x_{j}, \eta^{*}\left(r_{j}\right)\right)$. We apply Lemma 1 with $x_{0}=x_{j}, R=\eta^{*}\left(r_{j}\right)$ and $r=r_{j}$. Let $\rho_{j}=\operatorname{dist}\left(x, B\left(x_{j}, r_{j}\right)\right)$. We have

$$
\begin{aligned}
K * \mu_{j}^{\prime}(x) & \geq \int_{B\left(x, \rho_{j}\right) \cap B\left(x_{j}, \eta^{*}\left(r_{j}\right)\right)} K(x-y) d \mu_{j}^{\prime}(y) \\
& \geq K\left(\rho_{j}\right) \mu_{j}^{\prime}\left(B\left(x, \rho_{j}\right) \cap B\left(x_{j}, \eta^{*}\left(r_{j}\right)\right)\right) \\
& =K\left(\rho_{j}\right)\left\|\mu_{j}\right\| \frac{\left|B\left(x, \rho_{j}\right) \cap B\left(x_{j}, \eta^{*}\left(r_{j}\right)\right)\right|}{\left|B\left(x_{j}, \eta^{*}\left(r_{j}\right)\right)\right|} \\
& \geq A K\left(\rho_{j}\right)\left\|\mu_{j}\right\| .
\end{aligned}
$$

Obviously, $K * \mu_{j}(x) \leq K\left(\rho_{j}\right)\left\|\mu_{j}\right\|$, whence

$$
K * \mu_{j}(x) \leq A K * \mu_{j}^{\prime}(x) .
$$

Now suppose $x \notin \bigcup B\left(x_{j}, \eta^{*}\left(r_{j}\right)\right)$. Then (7) holds for all $j$. Hence we have from

$$
K * \mu(x)=\sum K * \mu_{j}(x) \leq A \sum K * \mu_{j}^{\prime}(x) \leq A K * \mu^{\prime}(x) \leq A .
$$

Suppose $x \in \bigcup B\left(x_{j}, \eta^{*}\left(r_{j}\right)\right)$, say $x \in B\left(x_{j_{0}}, \eta^{*}\left(r_{j_{0}}\right)\right)$ for some $j_{0}$. Then, by the disjointness of $\left\{B\left(x_{j}, \eta^{*}\left(r_{j}\right)\right)\right\}$ we have $x \notin \bigcup_{j \neq j_{0}} B\left(x_{j}, \eta^{*}\left(r_{j}\right)\right)$. Hence (6) and (7) yield

$$
\sum_{j \neq j_{0}} K * \mu_{j}(x) \leq A \sum_{j \neq j_{0}} K * \mu_{j}^{\prime}(x) \leq A K * \mu^{\prime}(x) \leq A .
$$

Since $\mu_{j_{0}}$ is an equilibrium measure, it follows that $K * \mu_{j_{0}} \leq 1$ on $\mathbb{R}^{N}$. Thus

$$
K * \mu(x)=K * \mu_{j_{0}}(x)+\sum_{j \neq j_{0}} K * \mu_{j}(x) \leq A
$$

Therefore (5) follows. The theorem is proved.

\section{Proof of Theorem 5}

First we prepare fundamental facts for $C_{K, p}$. We have the dual definition ([6, Theorem 14]). Let $\frac{1}{p}+\frac{1}{q}=1$.

Theorem B. Let $E$ be an analytic set. Then

$$
C_{K, p}(E)=\sup \left\{\|\mu\|^{p}: \mu \text { is concentrated on } E,\|K * \mu\|_{q} \leq 1\right\}
$$

Proof of Theorem 5. The proof will be carred out in a fashion similar to that of Theorem 3. It is sufficient to show that $\sum C_{K, p}\left(E \cap B\left(x_{j}, r_{j}\right)\right) \leq A C_{K, p}(E)$ in case the left hand side is positive. For simplicity we write $E_{j}$ for $E \cap B\left(x_{j}, r_{j}\right)$. Let $\varepsilon$ be an arbitrary number such that

$$
0<\varepsilon<\sum C_{K, p}\left(E_{j}\right)
$$


By Theorem B we can find a measure $\mu_{j}$ such that

$$
\begin{aligned}
& \mu_{j} \text { is concentrated on } E_{j}, \\
& \left\|K * \mu_{j}\right\|_{q}=1 \\
& C_{K, p}\left(E_{j}\right)-2^{-j} \varepsilon \leq\left\|\mu_{j}\right\|^{p} \leq C_{K, p}\left(E_{j}\right) .
\end{aligned}
$$

Let $\mu_{j}^{*}=C_{K, p}\left(E_{j}\right)^{1 / q} \mu_{j}$ and let $\mu^{*}=\sum \mu_{j}^{*}$. We observe that

$$
C_{K, p}\left(E_{j}\right)-2^{-j} \varepsilon \leq\left\|\mu_{j}^{*}\right\| \leq\left\|K * \mu_{j}^{*}\right\|_{q}^{q}=C_{K, p}\left(E_{j}\right) .
$$

We claim

$$
\left\|K * \mu^{*}\right\|_{q}^{q} \leq A \sum C_{K, p}\left(E_{j}\right)
$$

If we have (9), then

$$
\widetilde{\mu}=\left(\sum C_{K, p}\left(E_{j}\right)\right)^{-1 / q} \mu^{*}
$$

satisfies $\|K * \widetilde{\mu}\|_{q} \leq A$. Obviously $\widetilde{\mu}$ is concentrated on $E$ and by (8)

$$
\|\widetilde{\mu}\| \geq\left(\sum C_{K, p}\left(E_{j}\right)\right)^{-1 / q}\left(\sum C_{K, p}\left(E_{j}\right)-\varepsilon\right) .
$$

Since $\varepsilon$ is arbitrary, it follows from Theorem B that

$$
C_{K, p}(E) \geq A \sum C_{K, p}\left(E_{j}\right) .
$$

Thus the required inequality follows.

For the proof of (9) we put

$$
d \mu_{j}^{\prime}=\frac{\left\|\mu_{j}^{*}\right\|}{\left|B\left(x_{j}, \eta_{p}^{*}\left(r_{j}\right)\right)\right|} \chi_{B\left(x_{j}, \eta_{p}^{*}\left(r_{j}\right)\right)} d x
$$

and $\mu^{\prime}=\sum \mu_{j}^{\prime}$. By (8)

$$
\left\|\mu^{\prime}\right\|=\left\|\mu^{*}\right\| \leq \sum C_{K, p}\left(E_{j}\right)
$$

Let $d \mu^{\prime}=f d x$. Then, we observe from the disjointness of $B\left(x_{j}, \eta_{p}^{*}\left(r_{j}\right)\right)$ that

$$
f=\sum \chi_{B\left(x_{j}, \eta_{p}^{*}\left(r_{j}\right)\right)} \leq 1
$$

Hence by (10)

$$
\begin{aligned}
\left\|K * \mu^{\prime}\right\|_{q}^{q} & =\|K * f\|_{q}^{q} \leq\|K\|_{1}^{q}\|f\|_{q}^{q}=A\|f\|_{q}^{q} \\
& \leq A\|f\|_{\infty}^{q-1}\|f\|_{1} \leq A\|f\|_{1}=A\left\|\mu^{\prime}\right\| \leq A \sum C_{K, p}\left(E_{j}\right) .
\end{aligned}
$$

Let us compare $K * \mu^{*}$ and $K * \mu^{\prime}$. Applying Lemma 1, we obtain

$$
K * \mu_{j}^{*}(x) \leq A K * \mu_{j}^{\prime}(x) \text { for } x \notin B\left(x_{j}, \eta_{p}^{*}\left(r_{j}\right)\right) .
$$


Thus

$$
K * \mu^{*}(x) \leq A K * \mu^{\prime}(x) \text { for } x \notin \bigcup B\left(x_{j}, \eta_{p}^{*}\left(r_{j}\right)\right) .
$$

Suppose $x \in \bigcup B\left(x_{j}, \eta_{p}^{*}\left(r_{j}\right)\right)$, say $x \in B\left(x_{j_{0}}, \eta_{p}^{*}\left(r_{j_{0}}\right)\right)$ for some $j_{0}$. Then, by the disjointness of $\left\{B\left(x_{j}, \eta_{p}^{*}\left(r_{j}\right)\right)\right\}$ we have $x \notin \bigcup_{j \neq j_{0}} B\left(x_{j}, \eta_{p}^{*}\left(r_{j}\right)\right)$. Hence (12) yields

$$
K * \mu^{*}(x) \leq K * \mu_{j_{0}}^{*}(x)+A \sum_{j \neq j_{0}} K * \mu_{j}^{\prime}(x) \leq K * \mu_{j_{0}}^{*}(x)+A K * \mu^{\prime}(x) .
$$

It follows from (8) and (11) that

$$
\begin{aligned}
\left\|K * \mu^{*}\right\|_{q}^{q} & =\int_{\mathbb{R}^{N} \backslash \cup B\left(x_{j}, \eta_{p}^{*}\left(r_{j}\right)\right)}\left(K * \mu^{*}\right)^{q} d x+\int_{\bigcup B\left(x_{j}, \eta_{p}^{*}\left(r_{j}\right)\right)}\left(K * \mu^{*}\right)^{q} d x \\
& \leq A\left\|K * \mu^{\prime}\right\|_{q}^{q}+A \sum \int_{B\left(x_{j}, \eta_{p}^{*}\left(r_{j}\right)\right)}\left(K * \mu_{j}^{*}\right)^{q} d x \\
& \leq A \sum C_{K, p}\left(E_{j}\right)+A \sum\left\|K * \mu_{j}^{*}\right\|_{q}^{q} \\
& \leq A \sum C_{K, p}\left(E_{j}\right) .
\end{aligned}
$$

Therefore (9) follows. The theorem is proved.

\section{Proof of Theorem 2 And Theorem 4}

Let $C_{K, 1}(E)=C_{K}(E), \eta_{1}(r)=\eta(r), \eta_{1}^{*}(r)=\eta^{*}(r)$ and $\widetilde{E}_{K, 1}=\widetilde{E}_{K}$. Hereafter we let $1 \leq p<\infty$. Then we can prove Theorem 2 and Theorem 4 in the same way with the help of Theorem 3 and Theorem 5 .

Proof of Theorem 2 and Theorem 4. Take an arbitrary compact subset $F$ of $\widetilde{E}_{K, p}$. By the usual covering lemma we can find $x_{j} \in E$ such that

$$
\begin{aligned}
& F \subset \bigcup B\left(x_{j}, 5 \eta_{p}^{*}\left(r_{j}\right)\right), \\
& \left\{B\left(x_{j}, \eta_{p}^{*}\left(r_{j}\right)\right)\right\} \text { is disjoint, } \\
& r_{j}=\delta_{E}\left(x_{j}\right) .
\end{aligned}
$$

Let $E^{\prime}=\bigcup B\left(x_{j}, r_{j}\right)$. By definition this is a subset of $E$. We apply Theorem 3 and Theorem 5 for $B\left(x_{j}, r_{j}\right)$ and $E^{\prime}$. We obtain

$$
\sum C_{K, p}\left(B\left(x_{j}, r_{j}\right)\right) \leq A C_{K, p}\left(E^{\prime}\right) \leq A C_{K, p}(E) .
$$

On the other hand we have

$$
|F| \leq \sum\left|B\left(x_{j}, 5 \eta_{p}^{*}\left(r_{j}\right)\right)\right|=A \sum\left|B\left(x_{j}, \eta_{p}^{*}\left(r_{j}\right)\right)\right| .
$$

It follows from (2) and (4) that

$$
\left|B\left(0, \eta_{p}^{*}(r)\right)\right| \approx\left|B\left(0, \eta_{p}(r)\right)\right|=C_{K, p}(B(0, r)) .
$$

Hence

$$
|F| \leq A C_{K, p}(E)
$$

Since $F$ is an arbitrary compact subset of $\widetilde{E}_{K, p}$, the required inequality follows. The theorems are proved. 


\section{Generalization}

In this section we let $1 \leq p<\infty$ and use the convention for $C_{K, p}(E), \eta_{p}(r), \eta_{p}^{*}(r)$ and $\widetilde{E}_{K, p}$ as in the last section. Let $\Omega$ be a set in $\mathbb{R}_{+}^{N+1}$ with $\bar{\Omega} \cap \partial \mathbb{R}_{+}^{N+1}=\{0\}$. For simplicity we assume that $\Omega \supset\{(0, y): y>0\}$. Put $\Omega(y)=\{x:(x, y) \in \Omega\}$. We say that $\Omega$ satisfies the Nagel-Stein condition (abbreviated to (NS)), if

(i) $|\Omega(y)| \leq A y^{N}$ with $A=A(\Omega)$;

(ii) there is $\alpha>0$ such that

$$
\left(x_{1}, y_{1}\right) \in \Omega \text { and }\left|x-x_{1}\right|<\alpha\left(y-y_{1}\right) \Longrightarrow(x, y) \in \Omega \text {. }
$$

For $E$ we put

$$
\widetilde{E}_{K, p ; \Omega}=\bigcup_{x \in E}\left(x-\Omega\left(\eta_{p}^{*}\left(\delta_{E}(x)\right)\right)\right) .
$$

Theorem 6. Let $\Omega$ satisfy $(N S)$. Then

$$
\left|\widetilde{E}_{K, p ; \Omega}\right| \leq A C_{K, p}(E)
$$

where $A>0$ depends only on $N, K, p$ and $\Omega$.

Proof. The proof will be carried out in the same way as in the proof of Theorem 2 and Theorem 4 if we use the covering lemma due to Nagel-Stein [8, pp. 90-92]. But, for the completeness we give a proof.

Let $Q(y)=\bigcup_{x \in \Omega(y)} B(x, y)$. Obviously, $\Omega(y) \subset Q(y)$. In particular,

$$
\widetilde{E}_{K, p ; \Omega} \subset \bigcup_{x \in E}\left(x-Q\left(\eta_{p}^{*}\left(\delta_{E}(x)\right)\right)\right) .
$$

For a moment we fix $y>0$. By $\left[8, \operatorname{Lemma} 1\right.$ (d)] we find points $u_{1}, \ldots, u_{M} \in \Omega(y)$ so that $Q(y) \subset \bigcup_{j=1}^{M} B\left(u_{j}, 3 y\right)$. Note that the number $M$ is independent of $y$. We put

$$
\widetilde{Q}(y)=\bigcup_{j, k=1}^{M}\left\{u_{j}-u_{k}+\bigcup_{i=1}^{M} B\left(u_{i}, 9 y\right)\right\} .
$$

We observe that

$$
|\widetilde{Q}(y)| \leq A y^{N}
$$

where $A>0$ is independent of $y$.

Take an arbitrary compact subset $F$ of $\widetilde{E}_{K, p ; \Omega}$. We follow the same argument as in [8, pp. 90-92] and find points $x_{1}, \ldots, x_{J} \in E$ such that

$$
\begin{aligned}
& F \subset \bigcup_{j=1}^{J} x_{j}-\widetilde{Q}\left(y_{j}\right), y_{j}=\eta_{p}^{*}\left(\delta_{E}\left(x_{j}\right)\right) ; \\
& \left\{x_{j}-Q\left(y_{j}\right)\right\}_{j=1}^{J} \text { is disjoint. }
\end{aligned}
$$

We assumed that $\Omega \supset\{(0, y): y>0\}$ and hence $Q(y) \supset B(0, y)$. Hence, in particular, $\left\{B\left(x_{j}, y_{j}\right)\right\}_{j=1}^{J}$ is disjoint. Let

$$
E^{\prime}=\bigcup_{j=1}^{J} B\left(x_{j}, \delta_{E}\left(x_{j}\right)\right) .
$$


Obviously, $E^{\prime}$ is a subset of $E$. We apply Theorem 3 and Theorem 5 to $E^{\prime}$ to obtain

$$
\sum C_{K, p}\left(B\left(x_{j}, \delta_{E}\left(x_{j}\right)\right)\right) \leq A C_{K, p}\left(E^{\prime}\right) \leq A C_{K, p}(E)
$$

On the other hand, by (13), (14) and (15) we have

$$
|F| \leq A \sum y_{j}^{N}=A \sum\left|B\left(x_{j}, \eta_{p}^{*}\left(\delta_{E}\left(x_{j}\right)\right)\right)\right| \leq A \sum C_{K, p}\left(B\left(x_{j}, \delta_{E}\left(x_{j}\right)\right)\right) .
$$

Hence

$$
|F| \leq A C_{K, p}(E) .
$$

Since $F$ is an arbitrary compact subset of $\widetilde{E}_{K, p ; \Omega}$, the required inequality follows. The theorem is proved.

Remark 1. Let $\Omega$ be as in Theorem 6 . Then

$$
\left|\bigcup_{x \in E}\left(x+\Omega\left(\eta_{p}^{*}\left(\delta_{E}(x)\right)\right)\right)\right| \leq A C_{K, p}(E) .
$$

We record counterparts of Theorems $1-6$ for energy capacity. The energy capacity for the kernel $K$ is defined by

$$
e_{K}(E)=\sup \left\{\|\mu\|^{2}: \mu \text { is concentrated on } E, \int K * \mu d \mu \leq 1\right\} \text {. }
$$

We define $\eta_{e}(r)$ by

$$
\left|B\left(0, \eta_{e}(r)\right)\right|=e_{K}(B(0, r))
$$

and let $\eta_{e}^{*}(r)=\max \left\{\eta_{e}(r), 2 r\right\}$.

Theorem 7. Let $\Omega$ satisfy $(N S)$. Then

$$
\left|\bigcup_{x \in E}\left(x-\Omega\left(\eta_{e}^{*}\left(\delta_{E}(x)\right)\right)\right)\right| \leq A e_{K}(E) .
$$

where $A>0$ is independent of $E$. In particular,

$$
\left|\bigcup_{x \in E} B\left(x, \eta_{e}^{*}\left(\delta_{E}(x)\right)\right)\right| \leq A e_{K}(E) .
$$

Theorem 8. Suppose $\left\{B\left(x_{j}, \eta_{e}^{*}\left(r_{j}\right)\right)\right\}$ is disjoint and $E$ is an analytic subset of $\bigcup B\left(x_{j}, r_{j}\right)$. Then

$$
e_{K}(E) \leq \sum e_{K}\left(E \cap B\left(x_{j}, r_{j}\right)\right) \leq A e_{K}(E)
$$

where $A>0$ is independent of $E$.

We can prove Theorem 7 in exactly the same way as in the proof of Theorem 2 and Theorem 4, if we invoke Theorem 8 . The proof of Theorem 8 is somewhat a mixture of those of Theorem 3 and Theorem 5 . The details are left to the reader. 


\section{BOUNDARY BEHAVIOR OF HARMONIC FUNCTIONS}

In what follows we are interested in the boundary behavior of harmonic functions in $\mathbb{R}_{+}^{N+1}$. Hereafter we let $1 \leq p<\infty$. Following the idea in [2] and [5] we introduce the notion of thinness at the boundary. For a set $E \subset \mathbb{R}_{+}^{N+1}$ we put $E_{t}=\{(x, y): 0<y<t\}$ and $E^{*}=\bigcup_{(x, y) \in E} B(x, y)$. We recall that $B(x, y)$ is the $N$-dimensional ball with center at $x$ and radius $y$, so that the set $E^{*}$ is a set on the boundary $\mathbb{R}^{N}=\partial \mathbb{R}_{+}^{N+1}$. We shall combine the above notation and write

$$
E_{t}^{*}=\bigcup_{(x, y) \in E, 0<y<t} B(x, y) .
$$

Definition. Let $E \subset \mathbb{R}_{+}^{N+1}$. We say that $E$ is $C_{K, p}$-thin at $\partial \mathbb{R}_{+}^{N+1}$ if

$$
\lim _{t \rightarrow 0} C_{K, p}\left(E_{t}^{*}\right)=0 \text {. }
$$

Remark 2. If $E$ is $C_{K, p}$-thin at $\partial \mathbb{R}_{+}^{N+1}$, then the essential projection of $E$

$\{x:$ for any $t>0$ there is a positive number $y<t$ such that $(x, y) \in E\}$ is of $C_{K, p}$-capacity 0 , and hence of measure 0 .

We shall show the following theorems.

Theorem 9. Suppose $\Omega$ satisfies $(N S)$. Let

$$
\Omega_{K, p}=\left\{(x, y): x \in \Omega\left(\eta_{p}^{*}(y)\right)\right\} .
$$

If $E$ is $C_{K, p}$-thin at $\partial \mathbb{R}_{+}^{N+1}$, then

$$
\left|\bigcap_{t>0}\left\{x:\left(x+\Omega_{K, p}\right) \cap E_{t} \neq \emptyset\right\}\right|=0 .
$$

In other words, for almost all $x \in \partial \mathbb{R}_{+}^{N+1}, x+\Omega_{K, p}$ lies eventually outside $E$, i.e., there is $t=t_{x}>0$ such that $E_{t} \cap\left(x+\Omega_{K, p}\right)=\emptyset$.

Remark 3. It is not so difficult to see that $\eta_{p}^{*}(r) / r \rightarrow \infty$ as $r \rightarrow 0$ (cf. [1]). Hence $\Omega_{K, p}$ is a tangential region.

For a function $f$ on $\partial \mathbb{R}_{+}^{N+1}$ we denote by $P I(f)$ its Poisson integral. More precisely, let $\varphi(x)=A_{N}\left(|x|^{2}+1\right)^{-(N+1) / 2}$ and $\varphi_{y}(x)=y^{-N} \varphi(x / y)$, where $A_{N}>0$ is such that $\|\varphi\|_{1}=1$. Then we see that

$$
P I(f)(x, y)=\varphi_{y} * f(x) .
$$

Theorem 10. Let $\Omega \subset \mathbb{R}_{+}^{N+1}$ and suppose $\bar{\Omega} \cap \partial \mathbb{R}_{+}^{N+1}=\{0\}$. If $p=1$, then we assume that

$$
K(r) \approx r^{-N} \int_{0}^{r} K(t) t^{N-1} d t \text { for small } r>0
$$

Suppose $f \in L^{p}\left(\mathbb{R}^{N}\right)$. Then there is a set $E \subset \mathbb{R}_{+}^{N+1}$ such that $E$ is $C_{K, p}$-thin at $\partial \mathbb{R}_{+}^{N+1}$ and that

$$
\lim _{P \rightarrow x, P \in(x+\Omega) \backslash E} P I(K * f)(P)=K * f(x)
$$

for $C_{K, p}$-a.e. $x \in \partial \mathbb{R}_{+}^{N+1}$, i.e. there is a set $F \subset \partial \mathbb{R}_{+}^{N+1}$ such that $C_{K, p}(F)=0$ and (17) holds for $x \in \partial \mathbb{R}_{+}^{N+1} \backslash F$.

As a corollary to Theorem 9 and Theorem 10 we have the following theorem. This is a generalization of [7, Theorem 2.9]. 
Theorem 11. Let $\Omega \subset \mathbb{R}_{+}^{N+1}$ and suppose $\Omega$ satisfies $(N S)$. If $p=1$, then we assume that (16) holds. Suppose $f \in L^{p}\left(\mathbb{R}^{N}\right)$. Then

$$
\lim _{P \rightarrow x, P \in x+\Omega_{K, p}} P I(K * f)(P)=K * f(x)
$$

for almost all $x \in \partial \mathbb{R}_{+}^{N+1}$.

We note that Theorem 10 gives a sharp characterization of exceptional sets.

Proposition 1. Suppose $E$ is $C_{K, p}$-thin at $\partial \mathbb{R}_{+}^{N+1}$. Then there is $f \in L^{p}\left(\mathbb{R}^{N}\right)$ such that

$$
\inf _{(x, y) \in E, 0<y<t} P I(K * f)(x, y) \rightarrow \infty \text { as } t \rightarrow 0 .
$$

Finally we observe that the region $\Omega \subset \mathbb{R}_{+}^{N+1}$ in Theorem 10 is necessary. In fact, we have

Proposition 2. Let $g$ be an integrable function and let $x_{0} \in \partial \mathbb{R}_{+}^{N+1}$. Suppose there is a set $E$ such that the essential projection of $E$ is of measure 0 and

$$
\lim _{P \rightarrow x_{0}, P \notin E} P I(g)(P)=\alpha .
$$

Then

$$
\underset{x \rightarrow x_{0}}{\operatorname{ess} \lim _{x}} g(x)=\alpha .
$$

Remark 2 and Proposition 2 assert that if $h(P)=P I(K * f)(P)$ approaches a limit as $P$ approaches $x_{0} \in \partial \mathbb{R}_{+}^{N+1}$ outside a set $C_{K, p}$-thin at $\partial \mathbb{R}_{+}^{N+1}$, then $K * f$ must be essentially continuous at $x_{0}$. Unless a point has positive $C_{K, p}$-capacity, there exists $f \in L^{p}\left(\mathbb{R}^{N}\right)$ such that $K * f$ is essentially discontinuous at every point. In fact, assume that a point has null $C_{K, p}$-capacity. Let $E$ be a countable dense subset of $\mathbb{R}^{N}$. Then we see that $C_{K, p}(E)=0$, so that there is a nonnegative function $f \in L^{p}\left(\mathbb{R}^{N}\right)$ such that $K * f=\infty$ on $E$. Since $K * f$ is lower semicontinuous, it follows that ess $\lim \sup _{x \rightarrow x_{0}} K * f(x)=\infty$ for every $x_{0} \in \mathbb{R}^{N}$. At almost every point, $K * f$ is discontinuous even in the extended sense, since ess lim $\inf _{x \rightarrow x_{0}} K * f(x)<\infty$ for a.e. $x_{0} \in \mathbb{R}^{N}$ by the local integrability of $K * f$.

\section{Proof of Theorem 9}

We begin by an elementary geometrical observation.

Lemma 2. Let $\Omega \subset \mathbb{R}_{+}^{N+1}$ and suppose $\Omega(y)$ is increasing, i.e., if $y_{1}<y_{2}$, then $\Omega\left(y_{1}\right) \subset \Omega\left(y_{2}\right)$. Let $E \subset \mathbb{R}_{+}^{N+1}$ and let $E^{*}$ be as in Section 7 . Then

$$
\left\{x \in \mathbb{R}^{N}:(x+\Omega) \cap E \neq \emptyset\right\} \subset \bigcup_{x \in E^{*}}\left(x-\Omega\left(\delta_{E^{*}}(x)\right)\right),
$$

where $\delta_{E^{*}}(x)=\operatorname{dist}\left(x, E^{* c}\right)$.

Proof. We take an arbitrary point $x_{0}$ from the set in the left hand side of (21).

Then $\left(x_{0}+\Omega\right) \cap E \neq \emptyset$. Assume that $(x, y) \in\left(x_{0}+\Omega\right) \cap E$. This implies

(i) $x \in x_{0}+\Omega(y)$ and hence $x_{0} \in x-\Omega(y)$;

(ii) $(x, y) \in E$ and hence $B(x, y) \subset E^{*}$ by definition, so that $\delta_{E^{*}}(x) \geq y$. 
By the monotonicity of $\Omega(y)$ we have

$$
x_{0} \in x-\Omega\left(\delta_{E^{*}}(x)\right),
$$

which yields (21). The lemma is proved.

Proof of Theorem 9. We apply Lemma 2 for $\Omega_{K, p}$ replacing $\Omega$. Note that $\Omega_{K, p}(y)=$ $\Omega\left(\eta_{p}^{*}(y)\right)$. Then $(21)$ becomes

$$
\begin{aligned}
\left\{x \in \mathbb{R}^{N}:\left(x+\Omega_{K, p}\right) \cap E \neq \emptyset\right\} & \subset \bigcup_{x \in E^{*}}\left(x-\Omega_{K, p}\left(\delta_{E^{*}}(x)\right)\right) \\
& =\bigcup_{x \in E^{*}}\left(x-\Omega\left(\eta_{p}^{*}\left(\delta_{E^{*}}(x)\right)\right)\right) .
\end{aligned}
$$

Hence Theorem 6 yields

$$
\left|\left\{x \in \mathbb{R}^{N}:\left(x+\Omega_{K, p}\right) \cap E \neq \emptyset\right\}\right| \leq A C_{K, p}\left(E^{*}\right) .
$$

Apply this inequality for $E_{t}$ replacing $E$. Then the definition of thinness implies that

$$
\left|\left\{x:\left(x+\Omega_{K, p}\right) \cap E_{t} \neq \emptyset\right\}\right| \leq A C_{K, p}\left(E_{t}^{*}\right) \rightarrow 0 \text { as } t \rightarrow 0 .
$$

Thus the theorem follows.

$$
\text { 8. Proof of Theorem } 10 \text { In CASE } 1<p<\infty
$$

In this section we let $1<p<\infty$.

Lemma 3. Let $f$ be a nonnegative function in $L_{+}^{p}\left(\mathbb{R}^{N}\right)$ and let $\varepsilon>0$. Put

$$
\begin{aligned}
& E(f, \varepsilon)=\left\{(x, y) \in \mathbb{R}_{+}^{N+1}: P I(K * f)(x, y)>\varepsilon\right\} \\
& E^{*}(f, \varepsilon)=\bigcup_{(x, y) \in E(f, \varepsilon)} B(x, y) .
\end{aligned}
$$

Then

$$
C_{K, p}\left(E^{*}(f, \varepsilon)\right) \leq A\left(\frac{\|f\|_{p}}{\varepsilon}\right)^{p},
$$

where $A$ is independent of $f$ and $\varepsilon$.

Proof. Let

$$
E^{\prime}(f, \varepsilon)=\bigcup_{(x, y) \in E(f, \varepsilon)} B(x, y) \times\{y\} .
$$

Then the projection of $E^{\prime}(f, \varepsilon)$ is equal to $E^{*}(f, \varepsilon)$. Since $P I(K * f)$ is a positive harmonic function, it follows from the Harnack inequality that

$$
P I(K * f) \geq A \varepsilon \text { on } E^{\prime}(f, \varepsilon)
$$

where $A$ depends only on the dimension. Let us put

$$
F(x)=\sup _{y>0} \varphi_{y} * f(x)
$$


where $\varphi_{y}$ is the Poisson kernel as defined in Section 7. Then, the maximal inequality shows that

$$
\|F\|_{p} \leq A\|f\|_{p}
$$

([9, Theorem 3.7]). By definition

$$
K * F(x) \geq K *\left(\varphi_{y} * f\right)(x)=\varphi_{y} *(K * f)(x)=P I(K * f)(x, y) \text { for all } y>0 .
$$

Hence by $(22)$

$$
K * F \geq A \varepsilon \text { on } E^{*}(f, \varepsilon) .
$$

Thus, by (23),

$$
C_{K, p}\left(E^{*}(f, \varepsilon)\right) \leq A\left(\frac{\|F\|_{p}}{\varepsilon}\right)^{p} \leq A\left(\frac{\|f\|_{p}}{\varepsilon}\right)^{p}
$$

The lemma follows.

In [2] we proved a theorem similar to Theorem 10 by using oscillation. Here, we present a more direct proof without using oscillation. By $\mathbf{B}(P, r)$ we denote the $(N+1)$-dimensional open ball with center at $P$ and radius $r$.

Lemma 4. Let $f \in L^{p}\left(\mathbb{R}^{N}\right)$ and let $\delta>0$. Then there exist $E \subset \mathbb{R}_{+}^{N+1}$ and $F \subset \partial \mathbb{R}_{+}^{N+1}$ such that

(i) $C_{K, p}\left(E^{*}\right)<\delta$ and $C_{K, p}(F)<\delta$;

(ii) for each $\varepsilon>0$ there is $r>0$ such that

$$
\sup _{x \in \partial \mathbb{R}_{+}^{N+1} \backslash F}\left(\sup _{P \in \mathbf{B}(x, r) \cap \mathbb{R}_{+}^{N+1} \backslash E}|P I(K * f)(P)-K * f(x)|\right)<\varepsilon .
$$

Proof. In view of the definition of $C_{K, p}$ and Lemma 3, we can choose a sequence $g_{j} \in C_{0}^{\infty}\left(\mathbb{R}^{N}\right)$ such that

$$
\begin{aligned}
& C_{K, p}\left(F_{j}\right)<2^{-j} \delta \text { with } F_{j}=\left\{x:\left|K * g_{j}(x)-K * f(x)\right| \geq 2^{-j}\right\}, \\
& C_{K, p}\left(E_{j}^{*}\right)<2^{-j} \delta \text { with } E_{j}=\left\{P:\left|P I\left(K * g_{j}\right)(P)-P I(K * f)(P)\right| \geq 2^{-j}\right\} .
\end{aligned}
$$

Let $F=\bigcup_{j=1}^{\infty} F_{j}$ and $E=\bigcup_{j=1}^{\infty} E_{j}$. Then $C_{K, p}(F)<\delta$ and $C_{K, p}\left(E^{*}\right)<\delta$. Moreover we observe that

$$
\begin{aligned}
& K * g_{j} \rightarrow K * f \text { uniformly on } \partial \mathbb{R}_{+}^{N+1} \backslash F \\
& P I\left(K * g_{j}\right) \rightarrow P I(K * f) \text { uniformly on } \mathbb{R}_{+}^{N+1} \backslash E .
\end{aligned}
$$

Hence we can find $j_{0}$ such that

$$
\begin{aligned}
& \sup _{x \in \partial \mathbb{R}_{+}^{N+1} \backslash F}\left|K * g_{j_{0}}(x)-K * f(x)\right|<\frac{\varepsilon}{3}, \\
& \sup _{P \in \mathbb{R}_{+}^{N+1} \backslash E}\left|P I\left(K * g_{j_{0}}\right)(P)-P I(K * f)(P)\right|<\frac{\varepsilon}{3} .
\end{aligned}
$$


Since $P I\left(K * g_{j_{0}}\right)$ is uniformly continuous on $\mathbb{R}_{+}^{N+1}$ and has boundary values $K * g_{j_{0}}$, we can find $r>0$ such that

$$
\sup _{x \in \partial \mathbb{R}_{+}^{N+1}}\left(\sup _{P \in \mathbf{B}(x, r) \cap \mathbb{R}_{+}^{N+1}}\left|P I\left(K * g_{j_{0}}\right)(P)-K * g_{j_{0}}(x)\right|\right)<\frac{\varepsilon}{3} .
$$

The above three inequalities altogether imply (24). The lemma is proved

We prepare a geometrical observation for $\Omega$. We assume that $\bar{\Omega} \cap \partial \mathbb{R}_{+}^{N+1}=\{0\}$, which implies that for each $r>0$ there is $t>0$ such that

$$
\Omega \cap\{(x, y): 0<y<t\} \subset \mathbf{B}(0, r),
$$

or equivalently

$$
\Omega \backslash \mathbf{B}(0, r) \subset\{(x, y): y \geq t\} .
$$

Hence, if we have a decreasing sequence $r_{j} \downarrow 0$, then we can find $t_{j} \downarrow 0$ such that

$$
t_{j}<r_{j+1}
$$

and $\Omega \cap\left\{(x, y): 0<y<t_{j}\right\} \subset \mathbf{B}\left(0, r_{j+1}\right)$, or equivalently

$$
\Omega \backslash \mathbf{B}\left(0, r_{j+1}\right) \subset\left\{(x, y): y \geq t_{j}\right\} .
$$

Observe that

$$
\Omega \cap \mathbf{B}\left(0, r_{i}\right)=\bigcup_{j=i}^{\infty} \Omega \cap \mathbf{B}\left(0, r_{j}\right) \backslash \mathbf{B}\left(0, r_{j+1}\right) \subset \bigcup_{j=i}^{\infty} \mathbf{B}\left(0, r_{j}\right) \cap\left\{(x, y): y \geq t_{j}\right\}
$$

By translation we have for $x \in \partial \mathbb{R}_{+}^{N+1}$

$$
(x+\Omega) \cap \mathbf{B}\left(x, r_{i}\right) \subset \bigcup_{j=i}^{\infty} \mathbf{B}\left(x, r_{j}\right) \cap\left\{(x, y): y \geq t_{j}\right\} .
$$

Proof of Theorem 10. Take $\varepsilon_{j} \downarrow 0$. By Lemma 4 there are $E_{j} \subset \mathbb{R}_{+}^{N+1}, F_{j} \subset \partial \mathbb{R}_{+}^{N+1}$ and $r_{j} \downarrow 0$ such that

$$
\begin{aligned}
& \sum_{j} C_{K, p}\left(E_{j}^{*}\right)<\infty \text { and } \sum_{j} C_{K, p}\left(F_{j}\right)<\infty, \\
& \sup _{x \in \partial \mathbb{R}_{+}^{N+1} \backslash F_{j}}\left(\sup _{P \in \mathbf{B}\left(x, r_{j}\right) \backslash E_{j}}|P I(K * f)(P)-K * f(x)|\right)<\varepsilon_{j} .
\end{aligned}
$$

We choose $t_{j} \downarrow 0$ so that (25) and (26) hold. Let $E_{j}^{\prime}=E_{j} \cap\left\{(x, y): y \geq t_{j}\right\}$ and put $E=\bigcup_{j} E_{j}^{\prime}$. Then (27) yields

$$
C_{K, p}\left(E_{t_{i}}^{*}\right) \leq C_{K, p}\left(\bigcup_{j=i}^{\infty} E_{j}^{*}\right) \leq \sum_{j=i}^{\infty} C_{K, p}\left(E_{j}^{*}\right) \rightarrow 0 \text { as } i \rightarrow \infty
$$


so that $E$ is $C_{K, p}$-thin at $\partial \mathbb{R}_{+}^{N+1}$. Again by (27) we see that $F=\bigcap_{i=1}^{\infty} \bigcup_{j=i}^{\infty} F_{j}$ satisfies $C_{K, p}(F)=0$. Take $x \in \partial \mathbb{R}_{+}^{N+1} \backslash F$. We shall show that (17) holds. By definition there is $j_{0}=j_{0}(x)$ such that $x \notin F_{j}$ for all $j \geq j_{0}$. By (28)

$$
\sup _{P \in \mathbf{B}\left(x, r_{j}\right) \backslash E_{j}}|P I(K * f)(P)-K * f(x)|<\varepsilon_{j}
$$

for all $j \geq j_{0}$. Let $i \geq j_{0}$. In view of (26) and the definition of $E$

$$
\begin{aligned}
& \sup _{P \in(x+\Omega) \cap \mathbf{B}\left(x, r_{i}\right) \backslash E}|P I(K * f)(P)-K * f(x)| \\
& \leq \sup _{j \geq i}\left(\sup _{P \in \mathbf{B}\left(x, r_{j}\right) \cap\left\{(x, y): y \geq t_{j}\right\} \backslash E}|P I(K * f)(P)-K * f(x)|\right) \\
& \leq \sup _{j \geq i}\left(\sup _{P \in \mathbf{B}\left(x, r_{j}\right) \cap\left\{(x, y): y \geq t_{j}\right\} \backslash E_{j}}|P I(K * f)(P)-K * f(x)|\right) \\
& \leq \sup _{j \geq i} \varepsilon_{j}=\varepsilon_{i} \rightarrow 0 \text { as } i \rightarrow \infty \text {. }
\end{aligned}
$$

This implies (17). The theorem is proved.

\section{Proof of Theorem 10 In CASE $p=1$}

In this section we let $p=1$. In this case we cannot use the maximal inequality in the proof of Lemma 3. Instead, we estimate the kernel $K_{y}(x)=K * \varphi_{y}(x)$. Let

$$
\kappa(r)=K\left(\frac{r}{2}\right)+\frac{1}{r+1} r^{-N} \int_{0}^{r} K(t) t^{N-1} d t
$$

and let $\kappa(x)=\kappa(|x|)$ for $x \in \mathbb{R}^{N}$ as usual. The following lemma is a substitute of Lemma 3.

Lemma 5. Let $f \in L_{+}^{1}\left(\mathbb{R}^{N}\right)$ and let $\varepsilon>0$. Put

$$
\begin{aligned}
& E_{1}(f, \varepsilon)=\left\{(x, y) \in \mathbb{R}_{+}^{N+1}: 0<y<1, P I(K * f)(x, y)>\varepsilon\right\}, \\
& E_{1}^{*}(f, \varepsilon)=\bigcup_{(x, y) \in E_{1}(f, \varepsilon)} B(x, y) .
\end{aligned}
$$

Then

$$
C_{\kappa}\left(E_{1}^{*}(f, \varepsilon)\right) \leq A \frac{\|f\|_{1}}{\varepsilon},
$$

where $A$ is independent of $f$ and $\varepsilon$.

Proof. First we claim

$$
K_{y}(x) \leq A \kappa(x) \text { for } 0<y<1 .
$$


In fact we have

$$
\begin{aligned}
K_{y}(x)= & A_{N} \int_{\mathbb{R}^{N}} K(x-z) \frac{y}{\left(|z|^{2}+y^{2}\right)^{(N+1) / 2}} d z \\
\leq & A_{N} \int_{|x-z| \geq|x| / 2} K\left(\frac{|x|}{2}\right) \frac{y}{\left(|z|^{2}+y^{2}\right)^{(N+1) / 2}} d z \\
& \quad+A_{N} \int_{|x-z|<|x| / 2} K(x-z) \frac{y}{\left((|x| / 2)^{2}+y^{2}\right)^{(N+1) / 2}} d z \\
\leq & K\left(\frac{|x|}{2}\right)+A \frac{1}{|x| / y+1} \frac{1}{|x|^{N}} \int_{|z|<|x| / 2} K(z) d z \\
\leq & A \kappa(x) .
\end{aligned}
$$

In view of the claim and the Harnack inequality we have

$$
\kappa * f(x) \geq A \varepsilon \text { on } E_{1}^{*}(f, \varepsilon),
$$

whence the required inequality follows.

We observe that if $(16)$ holds, then $\kappa(r) \approx K(r)$ for small $r>0$, so that

$$
C_{K}(E) \approx C_{\kappa}(E) \text { for } E \subset U
$$

where $U$ is a bounded set. From this fact and Lemma 5 we can prove Theorem 10 in the same way as in Section 8 with a slight modification.

\section{Proof of Proposition 1 and Proposition 2}

Proof of Proposition 1. By definition there is $t_{j} \downarrow 0$ such that

$$
C_{K, p}\left(E_{t_{j}}^{*}\right)<2^{-j}
$$

We find nonnegative functions $f_{j} \in L^{p}\left(\mathbb{R}^{N}\right) \cap C_{0}^{\infty}\left(\mathbb{R}^{N}\right)$ such that

$$
K * f_{j} \geq 1 \text { on } E_{t_{j}}^{*}
$$

and $\left\|f_{j}\right\|_{p}^{p}<2^{-j}$. Let $f=\sum f_{j}$. Then $f \in L^{p}\left(\mathbb{R}^{N}\right)$. We show that this $f$ satisfies (18). Since $K * f_{j}$ is continuous, it follows that $P I\left(K * f_{j}\right)$ has continuous boundary values and

$$
P I\left(K * f_{j}\right) \geq 1 \text { on } E_{t_{j}}^{*} .
$$

Observe that if $(x, y) \in E_{t_{j}}$, then $B(x, y) \subset E_{t_{j}}^{*}$. Since the harmonic measure of $B(x, y)$ evaluated at $(x, y)$ is greater than a positive constant $A$ depending only on the dimension, it follows that

$$
P I\left(K * f_{j}\right) \geq A \text { on } E_{t_{j}} .
$$

Therefore

$$
P I(K * f) \geq A j \text { on } E_{t_{j}} .
$$

This implies (18). The proposition is proved. 
Proof of Proposition 2. We prove the proposition by contradiction. Suppose (20) does not hold. Then there is $\varepsilon_{0}>0$ such that

$$
\left|\left\{x \in B\left(x_{0}, r\right):|g(x)-\alpha|>2 \varepsilon_{0}\right\}\right|>0
$$

for all $r>0$. By (19) we see that there is $r_{0}>0$ such that

$$
|P I(g)(P)-\alpha|<\varepsilon_{0} \text { for } P \in B\left(x_{0}, r_{0}\right) \cap \mathbb{R}_{+}^{N+1} \backslash E .
$$

Let us consider the boundary values of $P I(g)$ on $B\left(x_{0}, r_{0}\right)$. It is well known that $P I(g)$ has nontangential boundary values $g$ a.e. on $\partial \mathbb{R}_{+}^{N+1}$. Hence, by (29), we can find a set $F \subset B\left(x_{0}, r_{0}\right)$ such that $|F|>0$ and

$$
\left|\operatorname{nt}_{P \rightarrow x} P I(g)(P)-\alpha\right|>2 \varepsilon_{0} \text { for } x \in F,
$$

where nt $\lim _{P \rightarrow x} P I(g)(P)$ means the nontangential limit of $P I(g)$ at $x$. In particular, there is a positive function $t(x)$ on $F$ such that

$$
|P I(g)(x, y)-\alpha|>\varepsilon_{0} \text { for } x \in F \text { and } 0<y<t(x),
$$

or, in other words

$$
|P I(g)-\alpha|>\varepsilon_{0} \text { on } A=\bigcup_{x \in F} I(x),
$$

where $I(x)=\{x\} \times(0, t(x))$. By (30) we see that $A \cap B\left(x_{0}, r_{0}\right) \subset E$. Obviously, $F$ is the essential projection of $A \cap B\left(x_{0}, r_{0}\right)$, Hence the essential projection of $E$ has positive measure. This is a contradiction.

\section{REFERENCES}

[1] H. Aikawa, Comparison of $L^{p}$-capacity and Hausdorff measure, Complex Variables 15 (1990), 223-232. MR 91i:31005

[2] H. Aikawa, Thin sets at the boundary, Proc. London Math. Soc. (3) 65 (1992), 357-382. MR 93g:31012

[3] H. Aikawa, Quasiadditivity of Riesz capacity, Math. Scand. 69 (1991), 15-30. MR 93d:31007

[4] H. Aikawa, Quasiadditivity of capacity and minimal thinness, Ann. Acad. Sci. Fenn. Ser. A. I. Mathematica 18 (1993), 65-75. MR 94d:31004

[5] A. A. Borichev, Boundary behavior in Dirichlet-type spaces, Uppsala University Department of Mathematics Report (1994), no. 3.

[6] N. G. Meyers, A theory of capacities for potentials of functions in Lebesgue classes, Math. Scand. 26 (1970), 255-292. MR 43:3474

[7] A. Nagel, W. Rudin and J. H. Shapiro, Tangential boundary behavior of functions in Dirichlettype spaces, Ann. of Math. 116 (1982), 331-360. MR 84a:31002

[8] A. Nagel and E. M. Stein, On certain maximal functions and approach regions, Adv. in Math. 54 (1984), 83-106. MR 86a:42046

[9] E. M. Stein and G. Weiss, Introduction to Fourier analysis on eulidean spaces, Princeton Univ. Press, 1970. MR 46:4102

Department of Mathematics, Faculty of Science, Kumamoto University, Kumamoto 860 , JAPAN

Current address: Department of Mathematics and Computer Science, Shimane University, Matsue 690, Japan

E-mail address: haikawa@shimane-u.ac.jp

Department of Mathematics, Uppsala University, Box 480, S-751 06 Uppsala, Sweden

E-mail address: borichev@math.uu.se 